\title{
CLASS NUMBERS OF TOTALLY IMAGINARY QUADRATIC EXTENSIONS OF TOTALLY REAL FIELDS
}

BY

\author{
JUDITH S. SUNLEY(1)
}

ABSTRACT. Let $K$ be a totally real algebraic number field. This paper provides an effective constant $C(K, h)$ such that every totally imaginary quadratic extension $L$ of $K$ with $h_{L}=h$ satisfies $\left|d_{L}\right|<C(K, h)$ with at most one possible exception.

This bound is obtained through the determination of a lower bound for $L(1, \boldsymbol{x})$ where $\boldsymbol{x}$ is the ideal character of $K$ associated to $L$. Results of Rademacher concerning estimation of $L$-functions near $s=1$ are used to determine this lower bound. The techniques of Tatuzawa are used in the proof of the main theorem.

I. Introduction. Let $K$ be an algebraic number field. The class number, $b_{K}$, of $K$ is one of the most important invariants of $K$. For example, the ring of integers, $\mathcal{O}_{K}$, of $K$ is a unique factorization domain if and only if $b_{K}=1$. Moreover, the degree of the maximal unramified abelian extension of $K$ is precisely $b_{K}$. Because of the importance of the class number, the problem of determining the class number is of central interest in algebraic number theory.

In general the determination of the class number of an arbitrary algebraic number field is not an easy task. There exist formulae for determining the class numbers of real and imaginary quadratic fields, but the problem of determining the class number of an arbitrary number field is still beyond the scope of contemporary number theory.

Gauss, in his Disquisitiones aritbmeticae [4], did extensive computations of the class numbers of real and imaginary quadratic fields. His tables suggest the following conjectures:

I. There are exactly 9 imaginary quadratic fields of class number 1 , namely those with discriminants $d_{K}=-3,-4,-7,-8,-11,-19,-43,-67,-163$.

II. If $b$ is a given positive integer, there exist only finitely many imaginary quadratic fields of class number $b$.

III. There exist infinitely many real quadratic fields of class number 1 .

Received by the editors December 17, 1971.

AMS (MOS) subject classifications (1969). Primary 1065; Secondary 1068.

Key words and phrases. Number field, class number, imaginary quadratic extension.

(1) This research was partially supported by NSF Science Development Grant GU-2061, and is substantially comprised of the author's doctoral dissertation at the University of Maryland. 
After many years of investigation, conjectures I and II have been proven, but conjecture III is still open. Conjecture II follows easily from the theorem of Siegel [17] which asserts

$$
\log b_{K} \sim \log \left|d_{K}\right|^{1 / 2} \quad\left(\text { as }\left|d_{K}\right| \rightarrow \infty\right)
$$

where $\sim$ denotes the asymptotic equivalence. It might be hoped that conjecture I would follow directly from II, but the only known proofs of Siegel's theorem are noneffective. The best one can do in improving Siegel's results is the result of Tatuzawa [22] who generalized earlier results of Heilbronn and Linfoot [10] to show that

$$
\left|d_{K}\right| \leq 2100 b_{K}^{2} \log \left(13 b_{K}\right)
$$

for any imaginary quadratic field with at most one exception. The result of Heilbronn and Linfoot is the special case $b_{K}=1$ which implies, upon inspection of tables, that there are at most 10 imaginary quadratic fields of class number 1.

Conjecture I has recently been proven by Stark [18]. Stark's proof completely bypasses the result of Heilbronn and Linfoot and proves directly that there are precisely nine imaginary quadratic fields of class number one. The methods used by Stark to settle conjecture I do not apply directly to the determination of all imaginary quadratic fields of arbitrary class number $h$. However, in 1970, Baker [1], Goldstein [6], and Stark [19] reduced the determination of all imaginary quadratic fields of class number two to a finite computation. Precise numerical computations have since been carried out and the class number 2 problem is now completely settled with independent solutions by Stark and Baker. This paper proceeds in a slightly different direction in the generalization of known results about class numbers. Let $K$ be a totally real algebraic number field. Then there are only finitely many totally imaginary quadratic extensions $L$ of $K$ having a given class number. This is an immediate result of the Brauer-Siegel theorem and the fact that the free parts of the unit groups of $K$ and $L$ have the same rank (see Goldstein [5]). We will consider the following

Problem. Determine effectively all totally imaginary quadratic extensions of $K$ having a given class number.

This problem is extremely difficult as can be evidenced by the fact that even in the case $K=\mathbf{Q}$ it has been solved only for class numbers one and two. Therefore this work should only be considered as a first step in the solution of the problem. The main result is a generalization of Tatuzawa's theorem.

Main Theorem. Let $K$ be a fixed totally real algebraic number field and let $L$ be a totally imaginary quadratic extension of $K$ baving conductor $F_{L}$ and class number $h$. Then there exists an effectively computable constant $c=c(K, b)$ such that $N F_{L} \leq c$, with the possible exception of one field $L$. 
This result has a number of applications. To begin with, it can be used to give an effective classification of all fields $L$ having class number one in the case where $K$ is normal over $\mathbf{Q}$. This has recently been carried out by Goldstein [7]. In the same paper Goldstein shows that if $K$ is a real quadratic field whose fundamental unit has norm -1 , an effective classification of all fields $L$ having class number two can be obtained.

Throughout this paper, unless explicitly stated otherwise, $K$ is assumed to be a totally real algebraic number field of degree $n \geq 2$ with discriminant $d . \chi$ is the real ideal character of $K$ associated to some totally imaginary quadratic extension $L$ of $K$; it is primitive modulo the ideal $F ; k$ will denote $|N F \cdot d| . \zeta_{K}(s)$ is the Dedekind zeta function associated to $K ; \zeta(s)$ is the Riemann zeta function. $L(s, \chi)$ is the series associated to $K$ and $\chi$ given by $L(s, \chi)=\Sigma_{\mathfrak{X}} \chi(\mathscr{U}) / N \mathscr{U}{ }^{s}$.

The method of proof in this paper revolves on determining a lower bound for $L(1, \chi)$ and using this to obtain an upper bound for the class number of $L$. In order to determine the lower bound for $L(1, \chi)$ it is first necessary to determine an upper bound for $L(s, \chi)$ in some region about $s=1$. This is done by using estimates obtained by Rademacher developed by applying the Phragmén-Lindelöf results to the gamma function [14].

In my initial work on this problem, the upper bound for $L(s, \chi)$ was obtained through an effectivization of Landau's results on ideal character sums [12]. The result is the following:

Theorem 1.1. Let $\Pi(x)=\Sigma_{N \rrbracket \leq x} 1, \Pi(x, \chi)=\Sigma_{N} \chi_{\leq x} \chi^{(\mathfrak{U})}$. Then for $K$ any arbitrary algebraic number field of degree $n \geq 2$ and discriminant $d$,

$$
\begin{gathered}
|\Pi(x)-b \kappa x|<e^{28.2 n+5}(n+1)^{5(n+1) / 2}|d|^{1 /(n+1)} \log ^{n}|d| \cdot x^{(n-1) /(n+1)} \\
|\Pi(x, \chi)|<e^{28.2 n+6}(n+1)^{5 n / 2+7 / 2} k^{1 /(n+1)} \log ^{n} k \cdot x^{(n-1) /(n+1)}
\end{gathered}
$$

where $b_{\kappa}$ is the residue of $\zeta_{K}(s)$ at $s=1, \chi$ is an ideal character over $K$ primitive modulo $F$ and $k=|N F \cdot d|$.

The proof of this theorem is to be found in [20]. As it is long, and can be circumvented through the use of Rademacher's results, the proof is not given here. In any case, the constants yielded by this method were not as small as those now obtainable. The results of this paper have been reported in the Bulletin of the American Mathematical Society [21].

II. Preliminary steps.

Lemma 2.1. Let $K$ be an arbitrary algebraic number field of degree $n \geq 2$ and discriminant $d$ with $b_{\kappa}$ the residue of $\zeta_{K}(s)$ at $s=1$. Then $b_{K}<$ $2^{2 n} e^{1 / 2}(1.3)^{n+1} \log ^{n-1}\left|d_{K}\right|$. If $K$ is a totally real field, then $b K<2^{n} e^{1 / 2}(1.3)^{n+1} \log ^{n-1}\left|d_{K}\right|$. 
Proof. In the standard terminology, let

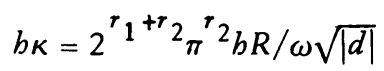

where $r_{1}$ is the number of real conjugates of $K, r_{2}$ is half the number of complex conjugates of $K, b$ is the class number of $K, R$ is the regulator of $K$, and $\omega$ is the number of roots of unity of $K$. From Landau [11, p. 74] we see for $s$ real and greater than one

$$
2^{r}{ }^{1} b R / \omega<A^{s} \Gamma(s / 2)^{r}{ }^{1} \Gamma(s)^{r} s^{n+1} /(s-1)^{n-1}
$$

where $A=|d|^{1 / 2} / 2^{r} \pi^{n / 2}$. In equation (1) set $s=1+1 / \log |d|$. Since $n \geq 2, s>1$. Clearly $A<|d|^{1 / 2}$. Then

$$
\begin{gathered}
\frac{2^{r}{ }^{1} H R}{\omega}<|d|^{1 / 2} e^{1 / 2}\left(\Gamma\left(\frac{1}{2}+\frac{1}{2 \log |d|}\right)\right)^{r}\left(\Gamma\left(1+\frac{1}{\log |d|}\right)\right)^{r} \\
\cdot\left(1+\frac{1}{\log |d|}\right)^{n+1} \log ^{n-1}|d| .
\end{gathered}
$$

This implies

$$
\begin{aligned}
b \kappa \leq & \frac{2^{r}{ }^{r} \pi^{r}}{d^{1 / 2}}|d|^{1 / 2} e^{i / 2}\left(\Gamma\left(\frac{1}{2}+\frac{1}{2 \log |d|}\right)\right)^{r}\left(\Gamma\left(1+\frac{1}{\log |d|}\right)\right)^{r} 2 \\
& \cdot\left(1+\frac{1}{\log |d|}\right)^{n+1} \log ^{n-1}|d| \\
< & (2 \pi)^{n} e^{1 / 2} 2^{n}\left(1+\frac{1}{\log 3}\right)^{n+1} \log ^{n-1}|d| \\
< & 2^{2 n} \pi^{n} e^{1 / 2}(1.3)^{n+1} \log ^{n-1}|d| .
\end{aligned}
$$

In case $K$ is totally real, $r_{1}=n, r_{2}=0$ and equation (2) yields $b \kappa<$ $2^{n} e^{1 / 2}(1.3)^{n+1} \log ^{n-1}|d|$.

Lemma 2.2. In the strip $-\eta \leq \sigma \leq 1+\eta ; 0<\eta \leq 1 / 2$, the Dedekind zeta function $\zeta_{K}(s)$ belonging to the arbitrary algebraic number field $K$ of degree $n$ and discriminant $d$ satisfies the inequality

$$
\left|\zeta_{K}(s)\right| \leq 3\left|\frac{1+s}{1-s}\right|\left(|d|\left(\frac{|1+s|}{2 \pi}\right)^{n}\right)^{(1+\eta-\sigma) / 2} \zeta(1+\eta)^{n} .
$$

Proof. See [14, p. 200].

Lemma 2.3. Let $K$ be a totally real field of degree $n \geq 2$ and discriminant $d$. Let $\chi$ be an ideal character over $K$ primitive modulo the ideal $F$. Then 


$$
|L(s, \chi)|<\zeta(1+\eta)^{n}\left\{\frac{k}{(2 \pi)^{n}} \prod_{q=1}^{n}|1+s|\right\}^{(1+\eta-\sigma) / 2}
$$

in the strip $-\eta \leq \sigma \leq 1+\eta, 0<\eta \leq 1 / 2$ where $k=|N F \cdot d|$.

Proof. Follows from [14, p. 204] and the fact that $\chi$ is an ideal character and therefore of finite order.

Theorem 2.4. Let $H(s)$ be regular for $\left|s-s_{0}\right| \leq r$, and $H\left(s_{0}\right) \neq 0$. Suppose $\left|H(s) / H\left(s_{0}\right)\right|<e^{M}$ in $\left|s-s_{0}\right| \leq r$ and suppose $H(s)$ bas no zeros in the right balf of this circle. Then

$$
-R \frac{H^{\prime}}{H}\left(s_{0}\right)+\sum_{\rho} R \frac{1}{s_{0}-\rho}<\frac{2 M}{r}\left(1+\left(2 \frac{r}{r_{0}} \log \frac{r}{r_{0}}\right)^{-1}\right)
$$

where $\rho$ runs over the zeros of $H(s)$ in $\left|s-s_{0}\right| \leq r_{0}<r$ counting multiplicities.

Proof. See Tatuzawa [22, p. 169].

III. A lower bound for $L(1, \chi)$. Let $F=\mathbf{Q}(\sqrt{-d})$ be an imaginary quadratic field of discriminant $-d$. Then the $L$-series $L(s, \chi)$ associated to $F$ is given by

$$
L(s, \chi)=\sum_{n=1}^{\infty} \chi^{(n) n^{-s}} \quad(\Re(s)>1)
$$

where $\chi^{(n)}=\left(\frac{-d}{n}\right)$ is the Kronecker symbol. A classical theorem of Dirichlet asserts that $L(1, \chi)=\pi b_{F} / \sqrt{d}$. Therefore, if one could establish a lower bound for $L(1, \chi)$ it would be possible to determine all those $F$ for which $b_{F} \leq b$. Such lower bounds were found by Landau and Hecke on the assumption of a weakened form of the Riemann hypothesis dealing with the real zeros of $L(s, \chi)$. Later Siegel [17] proved that, for $\epsilon>0^{\circ}$, there exists $c(\epsilon)$ such that $L(1, \chi)>$ $c(\epsilon) /|d|^{\epsilon}$. In fact, Siegel's proof is valid for any real Dirichlet character $\chi \neq \chi_{0}$. However, the constant $c(\epsilon)$ cannot be effectively computed using Siegel's methods. The essential achievement of Tatuzawa [22] is to compute $c(\epsilon)$ for all real Dirichlet characters with one possible exception. In this section we will generalize Tatuzawa's result.

Applying the decomposition of the Dedekind zeta function of $K$ and $L$ into a product of $L$-series to a well-known lemma [13, pp. 133-137] used in proving the Brauer-Siegel theorem, we find that for $\epsilon>0$, there exists $c(K, \epsilon)$ such that $L(1, \chi)>\dot{c}(K, \epsilon) / k^{\epsilon}$. As in the special case $K=\mathbf{Q}$, the constant $c(K, \epsilon)$ is ineffective. In this chapter we will effectively compute $c(K, \epsilon)$ for all real $\chi \neq \chi_{0}$ with one possible exception.

This section is divided into three parts. In the first, two functions are introduced which are of primary importance to the proof. Their Taylor and Dirichlet series expansions are then considered. In the second part, some information on 
the real zeros of these functions is obtained. The third part makes use of the information on the zeros to obtain the lower bound for $L(1, \chi)$.

3.1. The functions $F(s)$ and $G(s)$. Define

(1) $F(s)=\zeta_{K}(s) L(s, \chi)$;

(2) $G(s)=\zeta_{K}(s) L\left(s, \chi_{1}\right) L\left(s, \chi_{2}\right) L\left(s, \chi_{1} \chi_{2}\right)$;

where $\chi, \chi_{1}$ and $\chi_{2}$ are real ideal characters of the field $K$ determined by some totally imaginary quadratic extensions $L, L_{1}$, and $L_{2}$ of $K$ which are primitive modulo $F, F_{1}$, and $F_{2}$ respectively. It should be noted that both $F(s)$ and $G(s)$ have poles of order one at $s=1$ and are regular elsewhere.

We are particularly interested in the Dirichlet and Taylor series expansions for $F(s)$ and $G(s)$. Write $F(s)=\sum_{l=1}^{\infty} a_{l} l^{-s}$ for $\sigma>1$ and $F(s)=\sum_{m=0}^{\infty} a_{m}^{*}(2-s)^{m}$ for $|2-s|<1$. Then it is easily seen that $a_{m}^{*}=\sum_{l=1}^{\infty}\left(a_{l} \log ^{m} l\right) / l^{2} m$ !. Likewise writing $G(s)=\sum_{l=1}^{\infty} b_{l} l^{-s}$ for $\sigma>1$ and $G(s)=\sum_{m=0}^{\infty} b_{m}^{*}(2-s)^{m}$ for $|2-s|<1$, we find $b_{m}^{*}=\sum_{l=1}^{\infty}\left(b_{l} \log ^{m} l\right) / l^{2} m !$.

Lemma 3.1.1. In the above expansions, $a_{l} \geq 0$ and $b_{l} \geq 0$ for all l. Furthermore, if $l=(N)^{2 n}$ for $N \in \mathbf{Z}^{+}$, then $a_{l} \geq 1$ and $b_{l} \geq 1$.

Proof. The proof of this lemma is obtained by examining the Euler product representations of the Dedekind zeta function and the $L$-series which are valid for $\sigma>1$. In particular

$$
F(s)=\prod_{\Theta}\left(1-\frac{1}{N \& s}\right)^{-1}\left(1-\frac{\chi^{(\&)}}{N \Theta^{s}}\right)^{-1}
$$

and

$$
G(s)=\prod_{\&}\left(1-\frac{1}{N \mathbb{S}^{s}}\right)^{-1}\left(1-\frac{\chi_{1}^{(\&)}}{N \&^{s}}\right)^{-1}\left(1-\frac{\chi_{2}(\&)}{N \mathbb{S}^{s}}\right)^{-1}\left(1-\frac{\chi_{1} \chi_{2}(\&)}{N \&^{s}}\right)^{-1} .
$$

Let us examine the product for $G(s)$ for some given prime $\&$ under the possible conditions on $\chi_{1}$ and $\chi_{2}$. Let

$$
g(s, \&)=\left\{( 1 - N \& B ^ { - s } ) ( 1 - \chi _ { 1 } ( \& ) N B ^ { - s } ) \left(1-\chi_{2}(\&) N(B-s)\left(1-\chi_{1} \chi_{2}(\&) N(B-s)\right\}^{-1}\right.\right. \text {. }
$$

(1) $\chi_{1}{ }^{(\&)}=\chi_{2}{ }^{(\&)}=1$. Then

$$
g(s, \mathbb{B})=(1-N \&-s)^{-4}=\frac{1}{6} \sum_{m=0}^{\infty}(m+1)(m+2)(m+3) N \&-m s .
$$

(2) One of $\chi_{1}{ }^{(\&)}$ or $\chi_{2}{ }^{(\&)}$ is -1 and the other is either \pm 1 . Then

$$
g(s, \&)=(1-N \&-2 s)^{-2}=\sum_{m=0}^{\infty}(m+1) N(8-2 m s .
$$


(3) One of $\chi_{1}\left(\right.$ \&) or $\chi_{2}(\&)$ is 0 and the other is $1,-1$, or 0 . Then

$$
\begin{aligned}
& g(s, \text { \& })=\left(1-N(B-s)^{-2}=\sum_{m=0}^{\infty}(m+1) N(\&-m s,\right. \\
& g(s, \&)=\left(1-N(B-2 s)^{-1}=\sum_{m=0}^{\infty} N \&-2 m s,\right.
\end{aligned}
$$

or

$$
g(s, \mathbb{B})=(1-N \&-s)^{-1}=\sum_{m=0}^{\infty} N \&-m s,
$$

respectively. Thus in all cases,

$$
g(s, \mathbb{B})=\sum_{m=0}^{\infty} b(\&, m) N(\&-m s
$$

where $b(\mathbb{\&}, m) \geq 0$. Thus it is easily seen by examining $\Pi_{\Theta} g(s, \mathbb{B})$ that $b_{l} \geq 0$ for all $l$. Similarly it is easily shown that $a_{l} \geq 0$ for all $l$.

To prove the second part of the lemma it suffices to note that since $K$ has degree $n$ over $\mathbf{Q}$ any $l$ of the form $(N)^{n}$ is the norm of some ideal from $K$. Since in all the cases given above, any term of the form $(N \&)^{-2 s}$ has coefficient at least one, the $l$ th term in the Dirichlet series expansion has $a_{l} \geq 1$ (or alternatively $\left.b_{l} \geq 1\right)$ whenever $l=N^{2 n}$ for some $N \in \mathrm{Z}^{+}$. The above analysis in the case $K=\mathbf{Q}$ is due to Estermann [3].

This information about $a_{l}$ and $b_{l}$ allows us to make some estimates on partial sums in the Taylor series expansions for real $s$ such that $1 / 2 \leq s \leq 1$.

$$
\begin{aligned}
\sum_{m=0}^{r-1} a_{m}^{*}(2-s)^{m} & =\sum_{m=0}^{r-1} \sum_{l=1}^{\infty} \frac{a_{l} \log ^{m} l}{l^{2} m !}(2-s)^{m} \\
& \geq \sum_{m=0}^{r-1} \sum_{l=1}^{\infty} \frac{(2 n \log l)^{m}}{l^{4 n} m !}(2-s)^{m}=E(r, s) .
\end{aligned}
$$

Inequality (1) holds because of Lemma 3.1.1. Likewise, $\sum_{m=0}^{r-1} b_{m}^{*}(2-s)^{m} \geq E(r, s)$ for any positive integral $r$.

Lemma 3.1.2. For $1 / 2 \leq s \leq 1$, $s$ real,

$$
E(r, s) \geq \zeta(2 n s)-\frac{1}{(2 n s-1) q^{2 n s-1}}-\frac{\zeta(2 n s)-1}{\sqrt{2 r \pi}}\left(\frac{2 n e(2-s) \log q}{r}\right)^{r}
$$

for any positive integral $q$ and $r$. 
Proof.

$$
\begin{aligned}
E(r, s) & =\sum_{m=0}^{r-1} \sum_{l=1}^{\infty} \frac{(2 n \log l)^{m}}{l^{4 n} m !}(2-s)^{m} \\
& \geq 1+\sum_{l=2}^{q} \frac{\exp \{2 n(2-s) \log l\}}{l^{4 n}} \cdot\left\{1-\frac{1}{\sqrt{2 r \pi}} \frac{2 n e(2-s) \log q}{r}\right\}^{r} \\
& \geq \sum_{l=1}^{q} l^{-2 n s}-\left(\sum_{l=2}^{q} l^{-2 n s}\right)\left\{\frac{1}{\sqrt{2 r \pi}}\left(\frac{2 n e(2-s) \log q}{r}\right)^{r}\right\} \\
& \geq \zeta(2 n s)-\frac{1}{(2 n s-1) q^{2 n s-1}}-\frac{\zeta(2 n s)-1}{\sqrt{2 r \pi}}\left(\frac{2 n e(2-s) \log q}{r}\right)^{r}
\end{aligned}
$$

for any positive integers $q$ and $r$.

3.2. The zeros $F(s)$ and $G(s)$.

Proposition 3.2.1. Let $0<\epsilon<1 /(n+1)$, and let $k \geq e^{2}$. Suppose $L(1, \chi) \leq$ $.948 \epsilon / 4(n+1)^{2} b \kappa k^{\epsilon}$. Then $F(s)$ bas a real zero in $1-\epsilon / 4(n+1)^{2}<s<1$.

Proof. Consider the circle $C_{1}$ determined by $|s-2|=(n+2) /(n+1)$. On this circle $\sigma>(n-1) /(n+1)$. Take $\eta=1 / 2$ in Lemmas 1.2 and 1.3 . Then on the circle $C_{1}$,

$$
\begin{aligned}
|F(s)| & \leq \zeta\left(\frac{3}{2}\right)^{2 n}\left\{\frac{|\kappa d|}{(2 \pi)^{2 n}}|1+s|^{2 n}\right\}^{1 / 2+1 / 2(n+1)}\left|\frac{s+1}{s-1}\right|, \\
\left|\frac{b \kappa L(1, \chi)}{s-1}\right| & \leq \frac{2^{n} e^{1 / 2}(1.3)^{n+1}}{|s-1|} 3^{n}\left(\frac{1}{\pi}\right)^{n / 4} \log ^{n-1}|d| \cdot k^{1 / 4}
\end{aligned}
$$

by Lemmas 2.1, 2.2, and 2.3. Although the lemmas do not apply to all points on the circle, the points of largest absolute value for the functions lie within the range of application of the lemmas. Thus

$$
\begin{aligned}
& |F(s)-b \kappa L(1, \chi) /(s-1)| \\
& \quad \leq 8 \cdot 3^{2 n+1} e^{n / 3+1}(n+1)(2 / \pi)^{n / 4} \log ^{n-1}|d| \cdot k^{1 / 2+1 /(n+1)} .
\end{aligned}
$$

The function $F(s)-b \kappa L(1, \chi) /(s-1)$ is regular since $b \kappa$ is the residue of $\zeta_{K}(s)$ at $s=1$. Thus the inequality must also hold inside the circle $C_{1}$.

By Taylor's theorem, 


$$
F(s)-\frac{b \kappa L(1, \chi)}{s-1}=\sum_{m=0}^{\infty}\left(a_{m}^{*}-b \kappa L(1, \chi)\right)(2-s)^{m}
$$

for $|s-2|<1$. But since the left-hand side of the equation is regular, the Taylor series expansion on the right is valid for $|s-2|<2$. Thus by Cauchy's inequality,

$$
\begin{aligned}
\left|a_{m}^{*}-L(1, \chi) b \kappa\right| & \leq 8 \cdot 3^{n+1} e^{n / 3+1}(n+1)\left(\frac{2}{\pi}\right)^{n / 4} \log ^{n+1}|d| k^{1 / 2+1 /(n+1)}\left(\frac{n+1}{n+2}\right)^{m} \\
& =a_{1} \log ^{n-1}|d| \cdot k^{1 / 2+1 /(n+1)}\left(\frac{n+1}{n+2}\right)^{m}
\end{aligned}
$$

where $a_{1}=2^{n / 4+3} 3^{n+1} e^{n / 3+1}(n+1) \pi^{-n / 4}$.

Rewriting the above information, for $s$ real and $1-1 /(n+1)<s<1$,

$$
\begin{aligned}
F(s)> & \frac{L(1, \chi) b \kappa}{s-1}+\sum_{m=0}^{r-1} a_{m}^{*}(2-s)^{m}-b \kappa L(1, \chi) \sum_{m=0}^{r-1}(2-s)^{m} \\
& +\sum_{m=r}^{\infty}\left\{a_{m}^{*}-b \kappa L(1, \chi)\right\}(2-s)^{m} \\
> & \frac{L(1, \chi) b \kappa}{s-1}+\sum_{m=0}^{r-1} a_{m}^{*}(2-s)^{m}+b \kappa L(1, \chi) \frac{(2-s)^{r}-1}{s-1} \\
& -\left(a_{1} \log ^{n-1}|d| \cdot k^{1 / 2+1 /(n+1)}\right) \sum_{m=r}^{\infty}\left(\frac{2-s}{(n+2) /(n+1)}\right)^{m} \\
> & E(r, s)-\frac{b \kappa L(1, \chi)(2-s)^{r}}{1-s} \\
& -\left(a_{1} \log ^{n-1}|d| \cdot k^{1 / 2+1 /(n+1)}\right)(2-s)^{r}\left(\frac{n-1}{n+2}\right)^{r}\left(1-\frac{(2-s)(n+1)}{n+2}\right)^{-1}
\end{aligned}
$$

for any positive integer $r$.

In particular, for $s=1-\epsilon / 4(n+1)^{2}$,

$$
\begin{aligned}
F\left(1-\frac{\epsilon}{4(n+1)^{2}}\right)> & E\left(r, 1-\frac{\epsilon}{4(n+1)^{2}}\right)-\frac{b \kappa L(1, \chi)(2-s)^{r}}{1-s} \\
& -\left(a_{1} \log ^{n}|d| \cdot k^{1 / 2+1 /(n+1)}\right)(2-s)^{r}\left(\frac{n+1}{n+2}\right)^{r}\left(1-\frac{(2-s)(n+1)}{n+2}\right)^{-1}
\end{aligned}
$$

for all positive integral $r$. Take $r=\left[2^{2}(n+1)^{2} \log k\right]$, and take $q=\left[e^{2}\right]$ in the expression for $E(r, s)$. Then 


$$
\begin{aligned}
E\left(r, 1-\frac{\epsilon}{4(n+1)^{2}}\right) & >E(r, 1) \\
& >\zeta(2 n)-\frac{1}{(2 n-1) q^{2 n-1}}-\frac{\zeta(2 n)-1}{\sqrt{2 r \pi}}\left(\frac{2 n e(2-1) \log q}{r}\right)^{r} \\
& >1-\frac{1}{3 q^{3}}-\frac{\zeta(4)-1}{2(n+1) \sqrt{2 \pi \log k}}\left(\frac{2 n e \cdot 2}{2^{2}(n+1)^{2} \log k}\right)^{4(n+1)^{2} \log k} \\
& >1-\frac{1}{3 \cdot 7^{3}}-\frac{\zeta(4)-1}{2(n+1) \sqrt{2 \pi \log k}}\left(\frac{e}{(n+1) \log k}\right)^{4(n+1)^{2} \log k} \\
& >1-\frac{1}{3 \cdot 7^{3}}-\frac{.09}{2(n+1) \sqrt{2 \pi}}\left(\frac{e}{3}\right)^{36 \log k} \\
& >1-\frac{1}{1029}-\frac{.09}{2(n+1) \sqrt{2 \pi}}\left(\frac{e}{3}\right)^{36}>.998 .
\end{aligned}
$$

Moreover,

$$
\begin{aligned}
(2-s)^{r} & \leq(2-s)^{4(n+1)^{2} \log k} \\
& =\exp \left\{4(n+1)^{2} \log k \cdot \log (2-s)\right\}=k^{4(n+1)^{2} \log (2-s)}
\end{aligned}
$$

and

Therefore,

$$
\begin{aligned}
((n+1)) & (n+2))^{r}=\exp \{r \log ((n+1) /(n+2))\} \\
& \leq \exp \left\{\left(4(n+1)^{2} \log k-1\right) \log ((n+1) /(n+2))\right\} \\
& \leq(n+2) /(n+1) k^{4(n+1)^{2} \log ((n+1) /(n+2))}
\end{aligned}
$$

$$
\begin{aligned}
F & \left(1-\frac{\epsilon}{4(n+1)^{2}}\right) \\
> & .998-\frac{b \kappa L(1, \chi) k^{4(n+1)^{2} \log (2-s)}}{1-s} \\
& -a_{1} \log ^{n-1}|d| \cdot k^{1 / 2+1 /(n+1)}\left(\frac{n+2}{n-1}\right) k^{4(n+1)^{2}\{\log (2-s)+\log ((n+1) /(n+2))\}} \frac{(n+2)^{2}}{n+1} \\
> & .998-\left(\frac{b \kappa L(1, \chi) k^{\epsilon}}{\epsilon / 4(n+1)^{2}}\right) \\
& -a_{1} \frac{(n+2)^{3}}{(n+1)^{2}} \log ^{n-1}|d| \cdot k^{1 / 2+1 /(n+1)+4(n+1)^{2}\left\{\epsilon / 4(n+1)^{2}+\log ((n+1) /(n+2))\right\}} .
\end{aligned}
$$

Substituting in the value of $a_{1}$, we find 


$$
\begin{aligned}
& a_{1} \frac{(n+2)^{3}}{(n+1)^{2}} \log ^{n-1}|d| \cdot k^{1 / 2+1 /(n+1)+4(n+1)^{2}\left\{\epsilon / 4(n+1)^{2}+\log ((n+1) /(n+2))\right\}} \\
& \leq \exp \left\{\log a_{1}+(n-1) \log \log |d|+\left(\frac{1}{2}+\frac{1}{n+1}\right) \log k\right. \\
& \left.\quad+\left(\epsilon+4(n+1)^{2} \log \left(\frac{n+1}{n+2}\right)\right) \log k+\log \frac{(n+2)^{3}}{(n+1)^{2}}\right\} \\
& \leq \exp \left\{\log a_{1}+\log (n+2)+2 \log 1+\frac{1}{(n+1)}\right) \log k \\
& \left.\left.\quad+\frac{n-1}{2} \log |d|+\left(\frac{1}{2}+\frac{1}{n+1}+\epsilon+4(n+1)^{2} \frac{1}{2(n+1)^{2}}\right) \log +1\right) \log k\right\}
\end{aligned}
$$

$\leq \exp \{4.3+4.5 n+\{5 / 2+1 /(n+1)+(n-1) / 2+\epsilon-4(n+1)\} \log k\}$

$\leq \exp \{4.5(n+1)-(\overline{7 n} / 2-4 / 3) \log k\}$

$\leq \exp \{-2.5 n+1.8\}$ since $k>e^{2}$

$\leq \exp \{-3.2\}$.

Therefore,

$$
\begin{aligned}
F\left(1-\frac{\epsilon}{4(n+1)^{2}}\right) & >.998-\frac{4(n+1)^{2} L(1, \chi) k^{\epsilon} b \kappa}{\epsilon}-e^{-3.2} \\
& >.948-\frac{4(n+1)^{2} L(1, \chi) k^{\epsilon} b \kappa}{\epsilon} .
\end{aligned}
$$

If $L(1, \chi)<.948 \epsilon / 4(n+1)^{2} b \kappa k^{\epsilon}$, then $F(s)>0$. But $\lim _{s \rightarrow 1}-F(s)=-\infty$. Thus $F(s)$ must have a real zero in $1-\epsilon / 4(n+1)^{2}<s<1$ for all $0<\epsilon<1 /(n+1)$.

Proposition 3.2.2. For $0<\epsilon<1 /(n+1), \chi_{1}, \chi_{2}, \chi_{1} \chi_{2}$ nonprincipal, and $k_{1}$ $>e^{2}, k_{2}>e^{2}$

$$
G(s) \geq .948-b \kappa L\left(1, \chi_{1}\right) L\left(1, \chi_{2}\right) L\left(1, \chi_{1} \chi_{2}\right) k^{4(n+1)^{2}(1-s) /(1-s)}
$$

for real $s$ in the interval $1-\epsilon / 4(n+1)^{2}<s<1$.

Proof. As before, let $C_{1}$ be the circle $|s-2|=(n+2) /(n+1)$. Let $P=$ $L\left(1, \chi_{1}\right) L\left(1, \chi_{2}\right) L\left(1, \chi_{1} \chi_{2}\right)$. Then using Lemmas $2.1,2.2$, and 2.3 , on $C_{1}$,

$$
|G(s)| \leq 3 \zeta\left(\frac{3}{2}\right)^{4 n}\left|\frac{1+s}{1-s}\right|\left(k_{1} k_{2}\right)^{3 / 2-\sigma}|d|^{(3 / 2-\sigma) / 2}|1+s|^{n(3-2 \sigma)}(2 \pi)^{n(2 \sigma-3)} ;
$$

and 


$$
\left|\frac{b \kappa P}{s-1}\right| \leq \frac{2^{n} e^{1 / 2}(1.3)^{n+1} \log ^{n-1}|d| \zeta\left(3^{\prime} / 2\right)^{3 n} \pi^{-3 n / 4}\left(k_{1} k_{2}\right)^{1 / 2}}{|s-1|}
$$

Thus

$$
\begin{aligned}
& \left|G(s)-\frac{b \kappa P}{s-1}\right| \leq \frac{1}{|s-1|} \zeta\left(\frac{3}{2}\right)^{4 n}|1+s|^{n-2 n /(n+1)+1} 2^{n+1} \\
& \quad\left(k_{1} k_{2}\right)^{1 / 2+1 /(n+1)}|d|^{1 / 4+1 / 2(n+1)} \log ^{n-1}|d| ; \\
& \leq(n+1) 3^{4 n}|5 / 2|^{n-2 n /(n+1)+1} 2^{n+1}\left(k_{1} k_{2}\right)^{1 / 2+1 /(n+1)}|d|^{1 / 4+1 / 2(n+1)} \log ^{n}|d| .
\end{aligned}
$$

Employing the technique of Lemma 3.2.1 with $r=\left[4(n+1)^{2} \log \left(k_{1} k_{2}\right)\right]$ we obtain the desired result.

Proposition 3.2.3. $G(s)$ bas at most two zeros in the interval 1 $1 / 2^{4}(n+1)^{2} \log \left(k_{1} k_{2}\right)<s<1$ for $k_{1}, k_{2}>e^{2(n+1)}$ and $\chi_{1}, \chi_{2}$, and $\chi_{1} \chi_{2}$ not the principal character.

Proof. Let $s_{0}=1+1 / 2^{3}(n+1)^{2} \log \left(k_{1} k_{2}\right)$. Let the circle $C_{2}$ be given by $\left|s-s_{0}\right|=s_{0}-n /(n+1)$. Define the function

$$
H(s)=(s-1) \zeta_{K}(s) L\left(s, \chi_{1}\right) L\left(s, \chi_{2}\right) L\left(s, \chi_{1} \chi_{2}\right)=(s-1) G(s) .
$$

Then $H(s)$ is a regular function. On the circle $C_{2}$, using the Lemmas 2.1, 2.2, and 2.3,

(1) $\left|H(s) ! \leq 3 \zeta(3 / 2)^{4 n}\right| 1+s !\left(k_{1} k_{2}\right)^{3 / 2-\sigma}\left|d !^{(3 / 2-\sigma) / 2} \cdot\right| 1+\left.s\right|^{n(3-2 \sigma)}(2 \pi)^{n(2 \sigma-3)}$.

At the point $s_{0}$,

$$
\frac{1}{H\left(s_{0}\right)}=\frac{1}{\left|s_{0}-1\right|\left|\zeta_{K}\left(s_{0}\right)\right|\left|L\left(s_{0}, \chi_{1}\right)\right|\left|L\left(s_{0}, \chi_{2}\right)\right|\left|L\left(s_{0}, \chi_{1} \chi_{2}\right)\right|} .
$$

Recall the analysis of Lemma 3.1.1 which determined that the coefficients of the Dirichlet series for $G(s)$ were positive or zero. This implies that $G\left(s_{0}\right)>1$ since the coefficient of the first term is 1 and $s_{0}$ is real. Thus

$$
\begin{aligned}
& \left|\zeta_{K}\left(s_{0}\right) L\left(s_{0}, \chi_{1}\right) L\left(s_{0}, \chi_{2}\right) L\left(s_{0}, \chi_{1} \chi_{2}\right)\right|>1 \\
& \quad \Rightarrow 1 /\left|L\left(s_{0}, \chi_{1}\right) L\left(s_{0}, \chi_{2}\right) L\left(s_{.0}, \chi_{1} \chi_{2}\right)\right|<\zeta_{K}\left(s_{0}\right) .
\end{aligned}
$$

Putting this information together with (2), we find

$$
\frac{1}{\left|H\left(s_{0}\right)\right|} \leq \frac{\left|\zeta_{K}\left(s_{0}\right)\right|}{\left|s_{0}-1\right|\left|\zeta_{K}\left(s_{0}\right)\right|} \leq \frac{1}{\left|s_{0}-1\right|} \leq 2^{3}(n+1)^{2} \log \left(k_{1} k_{2}\right) \text {. }
$$


Combining inequalities (1) and (3) we have

$$
\begin{gathered}
\left|H(s) / H\left(s_{0}\right)\right| \leq 3 \zeta(3 / 2)^{4 n}|1+s|^{n(3-2 \sigma)+1}\left(k_{1} k_{2}\right)^{3 / 2-\sigma}|d|^{(3 / 2-\sigma) / 2} \\
(2 \pi)^{n(2 \sigma-3)} 8(n+1)^{2} \log \left(k_{1} k_{2}\right)
\end{gathered}
$$

for $s$ on $C_{2}$. Thus on this circle, by the methods of the preceding lemmas,

$$
\left|H(s) / H\left(s_{0}\right)\right| \leq \exp \left\{(15 / 4)(n+1) \log \left(k_{1} k_{2}\right)\right\}
$$

since $\log \left(k_{1} k_{2}\right)>4(n+1)$. Clearly $H(s)$ has no zeros in the right half of the interior of $C_{2}$. Therefore, applying Lemma 1.4, we find

$$
\begin{aligned}
& -\mathscr{R}\left(\frac{H^{\prime}}{H}\left(s_{0}\right)\right)+\sum_{\rho} R\left(\frac{1}{s_{0}-\rho}\right) \\
& \quad<\frac{2 \cdot(15 / 4)(n+1) \log \left(k_{1} k_{2}\right)}{s_{0}-n /(n+1)}\left(1+\left\{2 \frac{s_{0}-n /(n+1)}{r_{0}} \log \left(\frac{s_{0}-n /(n+1)}{r_{0}}\right)\right\}^{-1}\right)
\end{aligned}
$$

where $\rho$ runs over the zeros of $H(s)$ in $\left|s-s_{0}\right| \leq r_{0}<r$ counting multiplicities. Thus

$$
\begin{aligned}
-\frac{1}{s_{0}-1}+ & \left\{-\mathscr{R} \frac{\zeta_{K}^{\prime}}{\zeta_{K}}\left(s_{0}\right)-\mathfrak{R} \frac{L^{\prime}}{L}\left(s_{0}, \chi_{1}\right)-\mathscr{R} \frac{L^{\prime}}{L}\left(s_{0}, \chi_{2}\right)-\mathfrak{R} \frac{L^{\prime}}{L}\left(s_{0}, \chi_{1} \chi_{2}\right)\right\} \\
& +\sum_{\rho} R\left(\frac{1}{s_{0}-\rho}\right) \\
< & \frac{(30 / 4)(n+1) \log \left(k_{1} k_{2}\right)}{1 /(n+1)+1 / 2^{3}(n+1)^{2} \log \left(k_{1} k_{2}\right)} \\
& \left(1+\left(2 \frac{s_{0}-1 /(n+1)}{r_{0}} \log \left(\frac{s_{0}-1 /(n+1)}{r_{0}}\right)\right)^{-1}\right)
\end{aligned}
$$

Now $s_{0}$ is real and greater than one. Thus the quantity in brackets in inequality (4) is in fact positive. Therefore,

$$
\begin{aligned}
& -\frac{1}{s_{0}-1}+\sum_{\rho} R\left(\frac{1}{s_{0}-\rho}\right) \\
& \quad<\frac{(30 / 4)(n+1) \log \left(k_{1} k_{2}\right)}{s_{0}-n /(n+1)}\left(1+\left(2 \frac{s_{0}-n /(n+1)}{r_{0}} \log \left(\frac{s_{0}-n /(n+1)}{r_{0}}\right)\right)^{-1}\right)
\end{aligned}
$$


implies

$$
\begin{gathered}
\sum_{\rho} \mathfrak{R}\left(\frac{1}{s_{0}-\rho}\right) \\
H(\rho)=0 ;\left|s_{0}-\rho\right| \leq r_{0}<s_{0}-n /(n+1) \\
<\frac{1}{s_{0}-1}+\frac{(30 / 4)(n+1) \log \left(k_{1} k_{2}\right)}{s_{0}-n /(n+1)} \\
\cdot\left(1+\left(2 \frac{s_{0}-n /(n+1)}{r_{0}} \log \left(\frac{s_{0}-n /(n+1)}{r_{0}}\right)\right)^{-1}\right) \\
\leq \frac{30}{4}(n+1)^{2} \log \left(k_{1} k_{2}\right)\left(2+\left(2 \frac{s_{0}-n /(n+1)}{r_{0}} \log \left(\frac{s_{0}-n /(n+1)}{r_{0}}\right)\right)^{-1}\right) .
\end{gathered}
$$

Let

$$
r_{0}=\left(\frac{1}{2^{3}(n+1)}+\frac{1}{2^{4}(n+1)^{2}}\right) \frac{1}{\log \left(k_{1} k_{2}\right)}
$$

Then

$$
\begin{aligned}
\sum_{\rho} R\left(\frac{1}{s_{0}-\rho}\right) & <\frac{30}{4}(n+1)^{2} \log \left(k_{1} k_{2}\right)\left(2+\frac{3}{2^{5}(n+1) \log \left(k_{1} k_{2}\right)}\right) \\
& <16(n+1)^{2} \log \left(k_{1} k_{2}\right) .
\end{aligned}
$$

Suppose there were 3 zeros of $G(s)$ with $1 / 2^{4}(n+1)^{2} \log \left(k_{1} k_{2}\right)<\sigma<1$. Then

$$
\begin{aligned}
\sum_{\rho_{i}\left|s_{0}-\rho\right| \leq r_{0}} R\left(\frac{1}{s_{0}-\rho}\right) & >3\left\{\left(\frac{1}{2^{3}(n+1)^{2}}+\frac{1}{2^{4}(n+1)^{2}}\right) \frac{1}{\log \left(k_{1} k_{2}\right)}\right\}^{-1} \\
& >3\left\{\frac{3}{2^{4}(n+1)^{2} \log \left(k_{1} k_{2}\right)}\right\}^{-1}>16(n+1)^{2} \log \left(k_{1} k_{2}\right) .
\end{aligned}
$$

This is a contradiction to (5). Therefore there are at most two zeros of $G(s)$ with $\sigma$ in the desired interval.

3.3. The lower bound for $L(1, \chi)$.

Theorem 3.3.1. Let $\epsilon<\min \left\{1 /(n+1), 4(n+1)^{2} \alpha\right\}$, where $1-\alpha$ is the largest real zero of $\zeta_{K}(s)$. Assume that $\log k \geq \max \{2(n+1), 1 / \epsilon\}$. Then

$$
L(1, \chi)>.85 \epsilon / 4(n+1) 5^{n / 2} \kappa^{1 / 8+\epsilon} b \kappa
$$

except, possibly, for one exceptional character $\chi$. 
Proof. Suppose there exist $\chi_{1}$ and $\chi_{2}$ such that $L\left(1, \chi_{i}\right)<.85 \epsilon / 4(n+1) 5^{n / 2} k^{1 / 8+\epsilon} b \kappa$. Then by Proposition 3.2.1, $L\left(s, \chi_{1}\right)$ and $L\left(s, \chi_{2}\right)$ have real zeros in the interval $1-\epsilon / 4(n+1)^{2}<s<1$ since by hypothesis $\zeta_{K}(s)$ has no zeros in the interval.

Thus $G(s)$ has at least two real zeros counting multiplicities in $1-\epsilon / 4(n+1)^{2}<$ $s<1$. Let $s_{1}=1-1 /\left(2^{4}(n+1)^{2} \log k_{1} k_{2}\right)$ and $s_{2}=1-\epsilon / 4(n+1)^{2}$. Then with $P=L\left(1, \chi_{1}\right) L\left(1, \chi_{2}\right) L\left(1, \chi_{1} \chi_{2}\right)$,

$$
\begin{aligned}
\frac{b \kappa P\left(k_{1} k_{2}\right)^{4(n+1)^{2}\left(1-s_{1}\right)}}{1-s_{1}} & =b \kappa L\left(1, \chi_{1}\right) L\left(1, \chi_{2}\right) L\left(1, \chi_{1} \chi_{2}\right) \frac{\left(k_{1} k_{2}\right)^{4(n+1)^{2}\left(1-s_{1}\right)}}{1-s_{1}} \\
& <b \kappa L\left(1, \chi_{1}\right) L\left(1, \chi_{2}\right) 5^{n} \pi^{-n / 8}\left(k_{1} k_{2}\right)^{1 / 8} \frac{\left(k_{1} k_{2}\right)^{4(n+1)^{2}\left(1-s_{1}\right)}}{1-s_{1}}
\end{aligned}
$$

where the final inequality is obtained using Lemma 2.3 with $\eta=1 / 4$.

Then substituting the value of $s_{1}$, and noting $k_{i}>e^{1 / \epsilon}$,

$$
\begin{aligned}
& b \kappa P\left(k_{1} k_{2}\right)^{4(n+1)^{2}\left(1-s_{1}\right) /\left(1-s_{1}\right)} \\
& <b \kappa 2^{4}(n+1)^{2} 5^{n} \pi^{-n / 8} e^{1 / 4} \log \left(k_{1} k_{2}\right)\left(k_{1} k_{2}\right)^{1 / 8} L\left(1, \chi_{1}\right) L\left(1, \chi_{2}\right) \\
& <\left((.85)^{2} \log \left(k_{1} k_{2}\right) \epsilon^{2}\right) /\left(k_{1} k_{2}\right)^{\epsilon} b \kappa \text { by the hypothes is on } L\left(1, \chi_{1}\right) \text { and } L\left(1 ; \chi_{2}\right) \\
& <(.85)^{2} \cdot 4 /\left(e^{2} b \kappa \log \left(k_{1} k_{2}\right)\right)(\text { see }[22, \text { p. 177]) } \\
& <.85 \text {. }
\end{aligned}
$$

Thus $G\left(s_{1}\right)>0$. In a similar manner $G\left(s_{2}\right)>0$.

Now

$$
G(s) \geq .948-b \kappa P k^{4(n+1)^{2}(1-s) /(1-s)}
$$

and $G\left(s_{1}\right)$ and $G\left(s_{2}\right)$ are positive. Since the function $x^{\beta(1-s) /(1-s)}$ is convex for $\beta>0$ and $0<s<1, G(s)$ is concave in the interval $s_{1}<s<s_{2}$ and therefore must be positive throughout this interval. This implies that there are at least two real zeros of $G(s)$, counting multiplicities, to the right of $s_{2}$. If there were only two such zeros, this would imply $\lim _{s \rightarrow 1} G(s)>0$. But $\lim _{s \rightarrow 1}-G(s)=-\infty$. Thus we have a contradiction to the fact that there are only two zeros. There must be at least three zeros of $G(s)$ to the right of $s_{1}$. But this contradicts Proposition 3.2.3. Thus with at most one possible exception, for $k$ sufficiently large, $L(1, \chi)>.85 \epsilon / 4(n+1) 5^{n / 2} k^{1 / 8+\epsilon} b \kappa$. (Note this holds for any totally real algebraic number field $K$ with $\chi$ a real ideal character.)

IV. Generalization of the Heilbronn-Linfoot-Tatuzawa Theorem. In the preceding section, a lower bound for $L(1, \chi)$ is obtained with at most one exceptional 
$\chi$. This bound depends only on $k$ and parameters of the base field $K$. It now remains to relate this lower bound to the class number of the field $L$. This is accomplished by comparing the residues of $\zeta_{K}(s)$ and $\zeta_{L}(s)$ at $s=1$. Since $L$ is a quadratic extension of $K$, we know

$$
\zeta_{K}(s) L(s, \chi)=\zeta_{L}(s)
$$

Thus the residue at $s=1$ must be the same for both sides of (1); that is,

$$
\begin{aligned}
& \lim _{s \rightarrow 1^{+}}(s-1) \zeta_{K}(s) L(s, \chi)=\lim _{s \rightarrow 1^{+}}(s-1) \zeta_{L}(s) \\
& \Rightarrow b_{K^{K} K} L(1, \chi)=b_{L^{K}}{ }_{L}
\end{aligned}
$$

where

$$
b_{K} \kappa_{K}=2^{n} b_{K} R_{K} / w_{K} \sqrt{\left|d_{K}\right|} \text { and } b_{L} \kappa_{L}=2^{n} \pi^{n} b_{L} R_{L} / w_{L} \sqrt{\left|d_{L}\right|}
$$

Theorem 4.1. Let $1-\alpha$ be the largest real zero of $\zeta_{K}(s)$. Let $a_{3}=$ $10^{2.16 n+1.03}(n+1) \log ^{n-1}|d|$.

(a) If $a>1 /\left(8(n+1)^{2} \log a_{3}\right)$, then $k<\left(2 a_{3} \log a_{3} b_{L}\right)^{11 / 4}$, with at most one possible exceptional field $L$.

(b) If $a<1 /\left(8(n+1)^{2} \log a_{3}\right)$, then

$$
k<\max \left\{e^{1 / 4(n+1)^{2} \alpha} ;\left(a_{3} b_{L}\right)^{1 /\left(1-4(n+1)^{2}(n+2) \alpha\right)}\left(4(n+1)^{2} \alpha\right)^{-1 /\left(1-4(n+1)^{2}(n+2) \alpha\right)}\right\}
$$

with at most one possible exceptional field $L$.

Proof. Let us first make some general observations applying to both cases. Let $U_{K}$ (respectively $U_{L}$ ) denote the group of units of $K$ (respectively $L$ ) and let $W_{K}$ (respectively $W_{L}$ ) denote the group of roots of unity of $K$ (respectively $L)$. Then there is a natural injection $U_{K} / W_{K} \rightarrow U_{L} / W_{L}$. Set $\left[U_{L} / W_{L}: U_{K} / W_{K}\right]=$ $g_{0}(L / K)$. Since $L$ is totally imaginary and $K$ is totally real, $U_{L} / W_{L}$ and $U_{K} / W_{K}$ are free abelian groups of rank $n-1$, and therefore $g_{0}(L / K)$ is finite. An elementary argument shows that

$$
2^{n-1} R_{K}=g_{0}(L / K) R_{L}
$$

Moreover, elementary facts about the discriminants of algebraic number fields tell us that

$$
\left|d_{L}\right|=\left|N F \cdot d_{k}^{2}\right|
$$

Combining equations (2)-(5), we see

$$
L(1, \chi)=\frac{(2 \pi)^{n}}{g_{0}(L / K) w_{L}} \frac{b_{L}}{b_{K}} k^{-1 / 2}
$$




$$
\Rightarrow L(1, \chi) \leq(2 \pi)^{n} \frac{b_{L}}{b_{K}} k^{-1 / 2}
$$

This last estimate holds for all abelian characters $\chi$ which correspond to a totally imaginary quadratic extension of $K$. From $\$ 3$, we also have available a lower bound for $L(1, \chi)$ in this case. Namely, for $\epsilon \leq \min \left\{1 /(n+1), 4(n+1)^{2} \alpha\right\}$ and $\log k>\max \{2(n+1), 1 / \epsilon\}$,

$$
L\left(1, \lambda^{\prime}\right)>.85 \epsilon / 4(n+1) 5^{n / 2} k^{1 / 8+\epsilon} b \kappa .
$$

with at most one exception. Thus

$$
\frac{.85 \epsilon}{4(n+1) 5^{n / 2} k^{1 / 8+\epsilon} b K} \leq(2 \pi)^{n} \frac{{ }^{b} L}{b_{K}} k^{-1 / 2}
$$

$$
\begin{aligned}
& \Rightarrow k^{1 / 2-1 / 8-\epsilon} \leq(2 \pi)^{n}\left(b_{L} / b_{K}\right) 4(n+1) 5^{n / 2} b_{K} \kappa_{K} / .85 \epsilon \\
& \Rightarrow k^{3 / 8-\epsilon} \leq \frac{4(2 \pi)^{n}(n+1) 5^{n / 2} b_{L} / b_{K}}{.85 \epsilon} \cdot 2^{n} e^{1 / 2}(1.3)^{n+1} \log ^{n-1}|d| \leq a_{3} \epsilon^{-1} b_{L}
\end{aligned}
$$

using Lemma 2.1. The estimate in (8) is valid for $\epsilon \leq \min \left\{1 /(n+1), 4(n+1)^{2} \alpha\right\}$ and $\log k>\max \{2(n+1), 1 / \epsilon\}$ with at most one exception. It is now desirable to choose for $\epsilon$ a value which minimizes the upper bound for $k$. To do this we differentiate the two cases.

(a) If $\alpha \geq 1 /\left(8(n+1)^{2} \log a_{3}\right)$, then we may choose $\epsilon=1 /\left(2 \log a_{3}\right)$. In this case $\epsilon<1 /(n+1)$ and $\epsilon \leq 4(n+1)^{2} \alpha$. Moreover, $1 / \epsilon>2(n+1)$. Substituting this value for $\epsilon$ in (8) we have, for $k>a_{3}^{2}, k<\left(2 a_{3} \log a_{3} b_{L}\right)^{11 / 4}$ with at most one exception.

(b) If $\alpha<1 /\left(8(n+1)^{2} \log a_{3}\right)$ we use $\epsilon=4(n+1)^{2} \alpha$ to obtain the best possible result. Then for $k>e^{1 / 4(n+1)^{2} a}$,

$$
k<\left(a_{3} b_{L}\right)^{1 /\left(1-4(n+1)^{2}(n+2) a\right)}\left(4(n+1)^{2} \alpha\right)^{-1 /\left(1-4(n+1)^{2}(n+2) a\right)}
$$

with at most one exception.

This theorem can be restated in the following manner.

Theorem 4.2. Let $1-a$ be the largest real zero of $\zeta_{K}(s)$.

(a) If $\alpha>1 /\left(8(n+1)^{2} \log a_{3}\right)$, then all totally imaginary quadratic extensions $L$ of $K$ with class number $b_{L} \leq b_{0}$ are such that $k<\left(2 a_{3} \log a_{3} b_{0}\right)^{11 / 4}$ with at most one exceptional $L$.

(b) If $a<1 /\left(8(n+1)^{2} \log a_{3}\right)$, then all totally imaginary quadratic extensions $L$ of $K$ with class number $b_{L} \leq b_{0}$ are sucb that

$$
k<\max \left\{e^{1 / 4(n+1)^{2} a},\left(a_{3} b_{0}\right)^{1 /\left(1-4(n+1)^{2}(n+2) a\right)}\left(4(n+1)^{2} \alpha\right)^{-1 /\left(1-4(n+1)^{2}(n+2) \alpha\right)}\right\}
$$

with at most one possible exceptional $L$. 
Theorem 4.3. Let $K$ be a real quadratic field. Then

$$
k<\max \left\{e^{1 / 36 \beta},\left(a_{3} b_{L}\right)^{2 /(1-144 \beta)}(36 \beta)^{-3 /(1-144 \beta)}\right\}
$$

whenever $36 \beta<1 /(n+1)$, where

$\beta=6 \pi^{-1}\left|d_{K}\right|^{-1 / 2}\left\{1+6 \pi^{-1}\left|d_{K}\right|^{-1 / 2} \log \left|d_{K}\right|-12 \pi^{-1}\left|d_{K}\right|^{-1 / 2}\left(\log \log \left|a_{K}\right|+\pi / 2\right)\right\}$.

Proof. It can be shown (see Groswald $\left[8\right.$, p. 296]) that $\zeta_{K}(s)$ has no real zeros in $1-\beta<s<1$.

The following table gives the value of $C(K, 1)=\left(2 a_{3} \log a_{3}\right)^{11 / 4}$ where $K=$ $\mathbf{Q}(\sqrt{\theta})$ is a real quadratic field of class number 1 whose discriminant is less than 100. For all these fields, $\beta$ is too large to be of importance, thus the estimate of Theorem 4.1(a) applies.

V. Applications of the result.

5.1. Effective classification of all totally imaginary quadratic extensions $L$ with $h_{L}=b$ up to one possible exception. From the previous results, this effective classification is reduced to the problem of determining the class numbers of all totally imaginary quadratic extensions $L$ of $K$ such that $\left|d_{L}\right| \leq C(K, b)$.

There are several ways of doing this.

In the case of $L$ totally imaginary Minkowski's bound tells us that in every ideal class of $L$ there is an ideal $\mathscr{O}$ of $L$ such that $N \mathfrak{U} \leq(2 / \pi)^{n} \sqrt{\left|d_{L}\right|}$. Thus in order to determine all $L$ (with one possible exception) such that $b_{L}=b$, it suffices to examine those $L$ such that $: d_{L} \mid<C(K, b)$. For each of these $L$ one can determine all prime ideals $\&$ such that $N \&<(2 / \pi)^{n} \sqrt{\left|d_{L}\right|}$ and thus all integral ideals $\mathscr{U}$ such that $N \Re<(2 / \pi)^{n} \sqrt{\left|d_{L}\right|}$. These ideals can then be compared to determine which belong to the same class and thus to determine the class number of $K$. The exact technique which makes this process effective can be found in [2, Chapter 3].

This process of effectivization is extremely unwieldy and requires too many computations to be of much use. However, in certain cases class number formulas are available or at least conjectured.

Returning to the classical case, a formula has long been known for determining the class number of an imaginary quadratic field. Namely, for an imaginary quadratic field of discriminant $-d$ we have

$$
b=-\frac{w}{2 d} \sum_{(x, d)=1 ; 0<x<d} \chi(x) x .
$$

This formula has been the basis for all actual computation of class numbers of imaginary quadratic fields.

In the case where $K=\mathbf{Q}(\sqrt{D})$ is a real quadratic field, Hecke was able to obtain the following result. 


\begin{tabular}{|c|c|c|c|c|c|c|}
\hline$\theta$ & $d_{K}$ & $\sqrt{d_{K}}$ & $\log d_{K}$ & $a_{3}$ & $\log a_{3}$ & $C(K, 1)$ \\
\hline 2 & 8 & 2.83 & 2.08 & $1.40 \times 10^{6}$ & 14.15 & $7.792 \times 10^{20}$ \\
\hline 3 & 12 & 3.46 & 2.48 & $1.67 \times 10^{6}$ & 14.33 & $1.308 \times 10^{21}$ \\
\hline 5 & 5 & 2.24 & 1.61 & $1.08 \times 10^{6}$ & 13.89 & $3.664 \times 10^{21}$ \\
\hline 6 & 24 & 4.90 & 3.18 & $2.14 \times 10^{6}$ & 14.57 & $2.716 \times 10^{21}$ \\
\hline 7 & 28 & 5.29 & 3.33 & $2.24 \times 10^{6}$ & 14.62 & $3.110 \times 10^{21}$ \\
\hline 1 & 44 & 6.63 & 3.78 & $2.54 \times 10^{6}$ & 14.74 & $4.513 \times 10^{2}$ \\
\hline 3 & 13 & 3.61 & 2.56 & $1.72 \times 10^{6}$ & 14.36 & $1.436 \times 10^{2}$ \\
\hline 14 & 56 & 7.48 & 4.03 & $2.71 \times 10^{6}$ & 14.81 & $5.447 \times 10^{2}$ \\
\hline 17 & 17 & 4.12 & 2.83 & $1.90 \times 10^{6}$ & & $1.928 \times 10^{2}$ \\
\hline 19 & 76 & 8.72 & 4.28 & $2.87 \times 10^{6}$ & & $6.499 \times 10^{21}$ \\
\hline 21 & 21 & 4.58 & 3.04 & & 14.53 & \\
\hline 22 & 88 & 9.38 & 4.48 & $3.01 \times 10^{6}$ & 14.91 & \\
\hline 23 & 92 & 9.59 & 4.52 & $3.04 \times 10^{6}$ & 14.93 & $7.628 \times 10^{21}$ \\
\hline 29 & 29 & 5.38 & 3.37 & $2.26 \times 10^{6}$ & 14.63 & $3.221 \times 10^{21}$ \\
\hline 33 & 33 & 5.74 & 3.50 & $2.35 \times 10^{6}$ & 14.67 & $3.600 \times 10^{2}$ \\
\hline 37 & 37 & 6.08 & 3.61 & $2.42 \times 10^{6}$ & 14.70 & $3.943 \times 10^{2}$ \\
\hline 11 & 41 & 6.40 & 3.71 & $2.49 \times 10^{6}$ & 14.73 & $4.272 \times 10^{2}$ \\
\hline 53 & 53 & 7.28 & 3.97 & $2.67 \times 10^{6}$ & 14.80 & $5.212 \times 10^{2}$ \\
\hline 57 & 57 & 7.55 & 4.04 & $2.71 \times 10^{6}$ & 14.81 & $5.486 \times 10^{2}$ \\
\hline 61 & 61 & 7.81 & 4.11 & $2.76 \times 10^{6}$ & 14.83 & $5.770 \times 10^{2}$ \\
\hline 69 & 69 & 8.31 & 4.23 & $2.84 \times 10^{6}$ & 14.86 & $6.279 \times 10^{2}$ \\
\hline 73 & 73 & 8.54 & 4.29 & $2.88 \times 10^{6}$ & & $6.544 \times 10^{21}$ \\
\hline 77 & 77 & 8.89 & 4.34 & $2.91 \times 10^{6}$ & 14.89 & $6.770 \times 10^{21}$ \\
\hline 89 & 89 & 9.43 & 4.49 & $3.02 \times 10^{6}$ & 14.92 & $7.480 \times 10^{21}$ \\
\hline 93 & 93 & 9.64 & 4.53 & $3.04 \times 10^{6}$ & 14.93 & $7.678 \times 10^{21}$ \\
\hline & 97 & 9.85 & 4.57 & $3.67 \times 10^{6}$ & 14.94 & $7.878 \times 10^{2}$ \\
\hline
\end{tabular}

Table 
Theorem 5.1.1. Let $L=\mathbf{Q}(\sqrt{D}, \sqrt{\theta})$ be an imaginary quadratic extension of $K=\mathbf{Q}(\sqrt{D}), D>0$, with the integral ideal $F$ in $K$ as the conductor of $L$ over $K$, $\theta$ being a totally negative number in $K$. Then the class number $b_{L}$ of $L$ is given by

$$
b_{L}=b_{K} \frac{g_{0}(L / K)}{2^{n-1}} \frac{w_{L}}{w_{K}} \chi(\theta \gamma \sqrt{D}) \sum_{B} \bar{\chi}^{(B) G(B)}
$$

where $B$ runs over all the ray classes modulo $F$ in $K, \gamma \in K$ is chosen so that $(\gamma \sqrt{D})$ bas exact denominator $F$, and $\chi$ is the ideal character of $K$ associated to L. In this formula

$$
G(B)=v\left(\beta_{1}\right)\left\{\mathscr{P}_{2}\left(u_{B}\right) \frac{a+d}{c}-\sum_{l=0}^{c-1} \mathscr{P}_{1}\left(\frac{l+u_{B}}{c}\right) \mathscr{P}_{1} a\left(\frac{l+u_{B}}{c}-v_{B}\right)\right\}
$$

where $\left[\beta_{1}, \beta_{2}\right]$ is the basis of some ideal in $B, u_{B}=\operatorname{tr}\left(\beta_{1} \gamma\right), v_{B}=\operatorname{tr}\left(\beta_{2} \gamma\right)$, $v\left(\beta_{1}\right)=N\left(\beta_{1}\right) /\left|N\left(\beta_{1}\right)\right|$, and the rational integers $a, b, c$, and $d$ are determined by $\epsilon \beta_{2}=a \beta_{2}+b \beta_{1}, \epsilon \beta_{1}=c \beta_{2}+d \beta_{1}$, where $\epsilon$ is the generator of the group of units which are congruent to one modulo $f$ and totally positive. The functions $\mathcal{P}_{1}(x)$ and $\mathfrak{P}_{2}(x)$ are given by

$$
\begin{aligned}
& \mathcal{P}_{1}(x)=\left\{\begin{array}{cc}
x-[x]-1 / 2 & \text { for } x \notin Z \\
0 & \text { for } x \in Z
\end{array}\right\} \\
& \mathcal{P}_{2}(x)=1 / 2\left\{(x-[x])^{2}-(x-[x])\right\}+1 / 12 .
\end{aligned}
$$

Proof. See Siegel [16, p. 189] and Hecke [9].

It should be noted that $g_{0}(L / K)=1$ or 2 in this case and its exact determination takes only a finite number of steps. Moreover, all functions involved in the formula are elementary, $\mathscr{P}_{1}(x)$ and $\mathscr{P}_{2}(x)$ being symmetrized Bernoulli functions, and the formula is useful for computation purposes.

At the time the above result was obtained, Hecke [9, p. 2] conjectured a more general formula which would determine $b_{L} / b_{K}$ whenever $K$ is totally real and $L$ is a totally imaginary quadratic extension of $K$. Namely,

Conjecture 5.1.2. Es sei $k$ ein total reeller Körper, $\delta$ eine total (d.h. nebst sämtlichen Konjugierten) negative Zahl aus $k$. Dann bestimmt $\sqrt{\delta}$ einen Relativekörper $K$, welcher quadratisch bezüglich $k$ und total imaginär ist. Der Quotient der beiden Klassenzahlen $H$ and $b$ von $K$ bezw. $k$ ist dann als elementare arithmetische Funktionen von $\delta$ darstellbar, von analoger Beschaffenheit wie die rechte seite von (1).

Only a few cases are known where this conjecture has been proven. They include the two cases given above where $K=\mathbf{Q}$ or $K$ is a real quadratic field. The 
others were given by Kummer, where $K$ is the maximal real subfield of a cyclotomic field $\mathbf{Q}\left(\zeta_{p}\right)$ where $p$ is prime, and by Reidemeister in the case where $K$ is a totally real cubic field [15]. In these special cases the available formulae may be used to make the effectivization an easier process.

Thus we see that in any case, by performing suitable computations, we may classify all $L$ such that $b_{L}=b$ with at most one exception. It remains to see what can be said about the one possible exception.

5.2. $K$ is normal over $\mathbf{Q}$. In the case $K$ is normal over $\mathbf{Q}$ it is easily established that the exceptional field $L$ must also be normal over $\mathbf{Q}$. (See Goldstein [7].) Therefore we have

Theorem 5.2.1. Let $K$ be normal over $Q$; let $L$ be a totally imaginary quadratic extension of $K$ such that $b_{L}=b$. Then either

(1) $\left|d_{L}\right|<C(K, b)$; or

(2) $L$ is normal over $\mathbf{Q}$.

5.3. $K$ is normal over $\mathbf{Q}$, and $b_{L}=1$. In the special case where $b_{L}=1$, even more information is available. First of all, $b_{L}=1$ implies $b_{K}=1$ as well ([5], [7]). This limits the number of base fields one must investigate. In addition Goldstein [7] has shown that the exceptional field $L$ has a very special form.

Theorem 5.3.1. Let $K$ be a normal algebraic number field of class number one. Let $L$ be a totally imaginary quadratic extension of $K$ with $b_{L}=1$. Then either

(1) $\left|d_{L}\right|<C(K, 1)$;

(2) $F \mid 2$;

(3) $F \mid d_{K}$; or

(4) $L=K(\sqrt{\theta})$ where $\theta$ is the discriminant of some imaginary quadratic field of class number one.

Proof. Use Theorem 4.1 and the results of Goldstein [7].

Let us define a Siegel zero as any real zero of an ordinary Dirichlet $L$-series in the strip $0<\sigma<1$. A weak form of the Riemann hypothesis says there are no Siegel zeros.

Theorem 5.3.2. Let $K$ be a normal, totally real field of class number one. Assume that there are no Siegel zeros. Then for $\left|d_{K}\right|$ sufficiently large, $K$ can bave at most one totally imaginary quadratic extension $L$ of class number one; this $L$ is normal over $\mathbf{Q}$ and belongs to a finite, effectively determined set of fields.

Proof. If there are no Siegel zeros, case (a) of Theorem 4.1 applies. It should be noticed that this implies $N F \cdot\left|d_{K}\right|=O\left(\log ^{r}\left|d_{K}\right|\right)$ for some $r$ as $\left|d_{K}\right| \rightarrow \infty$. Then for $\left|d_{K}\right|$ sufficiently large (given $n$ ) we find $b_{L}=1 \Rightarrow N F<1$ with at most one exception. Goldstein's results can now be used to classify this one exception. 
Theorem 5.3.3. Let $K$ be any abelian extension of $\mathbf{Q}$ whose Galois group bas odd order and sucb that $b_{K}=1$. Then if $\left|d_{K}\right|$ is sufficiently large, there exists at most one totally imaginary quadratic extension $L$ of $K$ such that $b_{L}=1$. If $L$ exists, $L$ is normal over $\mathbf{Q}$ and belongs to a finite, effectively determined set of fields.

Proof. The classical result states that if $\chi$ is a Dirichlet character such that $\chi^{2} \neq \chi_{0}, L(s, \chi)$ has no real zeros for $1-1 /(c \log l)<s<1$ where $l$ is the period of $\chi$. This result can be effectivized by $c=1200$. If the Galois group of $K$ has odd order it has no elements of order 2 , and therefore $\zeta_{K}(s)$ has no real zeros in $1-1 /\left(1200 \log \left|d_{K}\right|\right)<s<1$. Therefore again case (a) of Theorem 4.1 applies.

5.4. $K=\mathbf{Q} \sqrt{D}$ and $b_{L}=2$. In this case additional information is available whenever the norm of the fundamental unit is -1 . This result is again due to Goldstein.

Theorem 5.4.1. Let $K=\mathbf{Q}(\sqrt{D})$ be a real quadratic field of class number one whose fundamental unit $\epsilon$ bas $N \epsilon=-1$. Then it is possible to effectively classify all $L$ for which $b_{L}=2$.

Proof. See Goldstein [7].

5.5. Points for further development. The results of this paper open up many possibilities for future research. The most fruitful area would appear to be the case where $K$ is a real quadratic field. Efforts are now being made to reduce the constants involved to a level where machine computation of all totally imaginary quadratic extensions of $K$ of class number one is possible. Moreover, using Goldstein's results [7] some information is available in certain cases for determination of the fields $L$ of class number two.

In particular, it would be desirable to find cases in which the one possible exception would not exist. One interesting question would be

Problem. Let $\mathcal{F}$ be the set of all totally imaginary biquadratic fields of class number one which contain a real quadratic field. Is $\mathcal{F}$ a finite set?

In order to answer this question, several improvements on the current results would be needed. First an improvement on the upper bound for the real zeros of $L(s, \chi)$ is needed where $\chi$ is a real Dirichlet character. Second, it would also be necessary to show that the exceptional field occurs for only a finite number of base fields $K$.

Another interesting application of the result is to the case of cyclotomic fields. Every cyclotomic field is a totally imaginary quadratic extension of its maximal totally real subfield. Moreover, this subfield is abelian over $\mathbf{Q}$. Thus another avenue of exploration is to determine those cyclotomic fields of class number one. 
Thus there are many approaches to future developments of the results of this paper. Further research in the determination of class numbers of imaginary quadratic fields is also important as these appear to play a decisive role in the relative case.

\section{BIBLIOGRAPHY}

1. Alan Baker, Imaginary quadratic fields of class number 2, Ann. of Math. (2) 94 (1971), 139-152.

2. Z. I. Borevič and I. R. Safarevič, Number theory, "Nauka", Moscow, 1964; English transl., Pure and Appl. Math., vol. 20, Academic Press, New York, 1966. MR 30 \#1080; MR $33 \# 4001$.

3. T. Estermann, On Dirichlet's L functions, J. London Math. Soc. 23 (1948), 275279. MR 10, 356 .

4. C. F. Gauss, Disquisitiones arithmeticae, Fleichser, Leipzig, 1870; English transl., Yale Univ. Press, New Haven, Conn., 1966。 MR 33 \#5545.

5. Larry Goldstein, A generalization of Stark's theorem, J. Number Theory 3 (1971), $323-346$.

6. - Imaginary quadratic fields of class number 2, J. Number Theory 4 (1972), 286-301.

7. - Relative imaginary quadratic fields of class number 1 or 2, Trans. Amer. Math. Soc. 165 (1972), 353-364.

8. Emil Grosswald, Negative discriminants of binary quadratic forms with one class in each genus, Acta Arith. 8 (1962/63), 295-306. MR 28 \#64.

9. E. Hecke, Bestimmung der Klassenzahl einer neuen Reihe von algebraischen Zahlkörpern, Göttinger Nachr. 1921.

10. H. Heilbronn and E. H. Linfoot, On the imaginary quadratic corpora of class number one, Quart. J. Math. Oxford Ser. 5 (1934), 293-301.

11. E. Landau, Einführung in die Elementare und Analytische Theorie der Algebraischen Zahlen und der Ideale, 2nd ed., Teubner, Leipzig, 1927.

12. - Verallgemeinerung eines Polyaschen Satzes auf algebrasche Zahlkörpern, Göttinger Nachr. 1918, 478-488. \#3974.

13. Serge Lang, Algebraic numbers, Addison-Wesley, Reading, Mass., 1964. MR 28

14. Hans A. Rademacher, On the Phragmén-Lindelöf theorem and some applications, Math. Z. 72 (1959/60), 192-204. MR 22 \#7982.

15. K. Reidemeister, Über die Relativklassenzahl gewisser relativquadratischer Zahlkörper, Abh. Math. Sem. Univ. Hamburg 1 (1921), 27-48.

16. C. L. Siegel, Lectures on advanced analytic number theory, Tata Institute of Fundamental Research, Bombay, 1961.

17. - Über die Klassenzahl quadratischer Zahlkörper, Acta Arith. 1 (1935), 275279.

18. Harold M. Stark, A complete determination of the complex quadratic fields of classnumber one, Michigan Math. J. 14 (1967), 1-27. MR 36 \#5102.

19. - Imaginary quadratic fields of class number 2, Ann. of Math. (2) 94 (1971), 153-174. 
20. J. Sunley, On the class numbers of totally imaginary quadratic extensions of totally real fields, Ph.D. Dissertation, University of Maryland, College Park, Md., 1971.

21. On the class numbers of totally imaginary quadratic extensions of totally real fields, Bull. Amer. Math. Soc. 78 (1972), 74-76.

22. T. Tatuzawa, On a theorem of Siegel, Japan. J. Math. 21(1951), 163-178. MR 14, 452.

DEPARTMENT OF MATHEMATICS AND STATISTICS, AMERICAN UNIVERSITY, WASHINGTON, D. C. 20016 